A rendere il volume qui esaminato interessante e persuasivo non è però solo la ricostruzione della presa di coscienza civile e politica del Consolo maturo ma anche l'analisi, attenta e dettagliata, delle scelte stilistico-espressive collegate a questa presa di coscienza. Francese mette in campo la sua solida conoscenza della produzione letteraria e non dello scrittore siciliano allo scopo di individuare quelle scelte che separano un testo come L'olivo e l'olivastro (1994) o molte delle storie comprese ne La mia isola è Las Vegas (libro uscito postumo, nel 2012), per citare due esempi significativi, dai principali romanzi e raccolte di racconti che li precedono - da Il sorriso dell'ignoto marinaio (1976) a Retablo (1987), da Le pietre di Pantalica (1988) a Nottetempo, casa per casa (1992). Ciò che si pone in risalto è che la narrativa dell'ultimo Consolo, sia che presenti un io narrante sia che si affidi alla terza persona, evidenzia una marcata e inedita impronta autobiografica. In una narrazione sperimentale qual è L'olivo e l'olivastro — narrazione che Consolo aveva denominato "poematica" in quanto tesa a scavalcare a ritroso la tradizione del romanzo per recuperare forme e modalità proprie dell'epica — tale impronta autobiografica si mescola con una pronunciata tendenza al frammentismo lirico e con un'altrettanto spiccata ricerca della parola che restituisce non tanto l'idea ma piuttosto l'immagine, ovvero della parola icastica.

In conclusione, va riconosciuto esplicitamente all'autore di questo volume un doppio merito: da un lato, un merito specifico, l'aver arricchito l'opera critica su uno scrittore importante e ancora in larga parte da scoprire come Consolo; dall'altro, un merito più generale, l'aver esplorato la complessità del rapporto fra letteratura e impegno sulla base di una disamina approfondita di un significativo caso particolare.

\title{
Luca Pocci
}

The University of Western Ontario

\section{Marialuigia Sipione e Matteo Vercesi, a cura di. Filologia ed Ermeneutica. Studi di letteratura italiana offerti dagli allievi a Pietro Gibellini. Brescia: Editrice Morcelliana. 2105. Pp. 347. ISBN 978-88-372-2883-5.}

Il volume nasce dalla volontà degli allievi, e collaboratori più stretti, del professor Pietro Gibellini di celebrare i suoi settant'anni con un "regalo che, da un lato, tenesse conto delle sue grandi passioni accademiche — la filologia e l'ermeneutica, ma più 
in generale la letteratura - e che, dall'altro, non tradisse la facilità affabulatoria rinvenibile in ogni suo contributo" (5). Gli autori degli scritti presenti nel volume cercano di rendere omaggio al maestro comune con contributi che offrono "uno specimen dei suoi interessi e filoni di ricerca" (6): dalla poesia dialettale del Sette al Novecento - Belli, Carducci, Pascoli, D’Annunzio - fino alla filologia d'autore e alla critica tematica. I testi pervenuti sono stati disposti in ordine cronologico alla luce della materia trattata, ma nello specifico il volume presenta una struttura tripartita.

Il primo nucleo verte su questioni filologiche. Maria Belponer, "Filologia e mitopoiesi tra le antologie latine di Pascoli e i Conviviali" (93-104), dedica una riflessione su Lyra e Conviviali, e alle interazioni fra le due opere, "attraverso il sapiente montaggio delle traduzioni che Pascoli diede di alcuni passi, soprattutto omerici" (94). Sara Campardo, "L'Elettra di D’Annunzio. Saggio di edizione critica" (155-168), si occupa della genesi di Elettra, secondo libro delle Laudi del cielo del mare della terra e degli eroi, dove Dante "è primo e un po' isolato dagli altri, tra gli eroi dell'arte" (155). Rosy Cupo, "Per l'edizione critica del Belmoro di Corrado Alvaro" (177-189), offre una "verifica preliminare delle stratificazioni redazionali dell'opera e una ricognizione sui tempi di composizione" (177) dell'opera di Alvaro. Donatella Fedele, "Schegge di estetica dusiana nelle lettere a D’Annunzio" (135-154), presenta l'epistolario di Eleonora Duse, focalizzando il discorso sulle lettere a D’Annunzio, "rimaste quasi del tutto inedite a causa della loro oscurità” (135). Danijela Maksimović Janjić, "Esempi di reciproche influenze lessicali fra Pascoli e D’Annunzio" (121-134), esamina i "rapporti conflittuali e proficui nel generare espressioni simili nelle parole e diversi nei concetti” fra i due autori (121). Sara Lucchetta, "Le redazioni del Plutone casareccio di Giorgio Vigolo. Un 'furore ossessivo'” (191-200), sofferma l'analisi sulla redazione formale del racconto il Plutone casereccio, presente nella raccolta La notti romane, "una delle opere più felici dello scrittore" (191). Maddalena Rasera, "Annotazioni sulle Colpe altrui di Grazia Deledda" (169-176), presenta la ricerca svolta su un "documento conservato nel fondo Hérelle, un fascicolo di fogli slegati, dattiloscritti, del romanzo Le colpe altrui" (169). Edoardo Ripari, "Nuove primizie e reliquie di Giosuè Carducci" (77-92), si occupa di alcuni juvenilia inediti che "testimoniano l'indubbia predisposizione alle lettere di un giovane poeta focoso e pensoso" (77). La sezione si conclude con Francesca Suppa, "Vicenda umana e vertigine cosmica. La guazza nelle carte pascoliane" (105-119), che presenta la genesi compositiva dell'opera di Pascoli. 
La seconda sezione affronta gli aspetti metodologici: Nicola di Nino, "De Sica e la polemica sociale. La scena della trattoria in Ladri di biciclette" (221227), propone un'interessante lettura di una scena chiave del cinema Neorealista attraverso uno dei "film che ha saputo maggiormente descrivere l'Italia dell'immediato secondo dopoguerra" (221); Stefano D’Ambrosio, "Sul romanzo storico postmoderno" (239-248), si sofferma sui romanzi storici che "per caratteristiche strutturali e nodi concettuali denunciano il proprio legame di dipendenza dal vasto dibattito sul concetto di postmodernità" (239); Elena De Bortoli, "La fonte biblica nelle Gerusalemme Conquistata" (39-48), offre un'analisi del poema incentrata sull'uso delle diverse fonti, classiche e scritturali; Valetina Puleo, "Sul mito di Orfeo ed Euridice in Gesualdo Bufalino" (249-258), ragiona sull'intertestualità mitologica nella prosa di Bufalino, "uno degli autori più autenticamente polytropoi: sa far incontrare paradigmi mitici con ambientazioni contemporanee" (249) ed infine Matteo Vercesi, "Gli studi sulla letteratura dialettale di Pietro Gibellini" (293-304), propone una valutazione, e un bilancio, sull'attenzione che Gibellini ha riservato al "fecondo bacino delle opere letterarie nei dialetti d'Italia" (293), radicata soprattutto dalla lezione degli ammirati maestri Isella, Contini e Dionisotti.

La terza sezione "raccoglie medaglioni monografici su un dato tema o uno specifico autore" (6). S'inizia da Concetta di Franza, "Gli animali nella poesia di Giovanni Raboni” (259-271), che pone l'attenzione sulla presenza, nella poetica raboniana, degli animali come "compromissione con la realtà, all'insegna di una inclusività e di una transitività" (259), per poi continuare con Laura Bolzonello, "Nel vivaio dell'indivisa vita condivisa'. Patrizia Valduga e Giovanni Raboni" (271-281), che propone una lettura incrociata dei due autori, legati dal doppio filo dell'amore e della ricerca poetica. Diletta Ferrai, "Giannetto Valzelli. L'arte delle parole, l'elogio delle cose" (229-238), invece, si sofferma sulla poliedrica figura di Valzelli: scrittore, giornalista, critico d'arte, studioso di storia e di letteratura, che animò la scena intellettuale bresciana per oltre cinquant'anni. Enrica Gambin, "La 'metafora medica' nei carmi in onore di Raffaello Sanzio" (29-38), introduce il rapporto fra pittura e poesia nel Cinquecento dalla straordinaria personalità di Raffaello. Elena Maiolini, “ 'Ora vien la grandine'. Appunti sull'ira nei Promessi Sposi" (49-57), affronta le diverse accezioni che l'ira assume nel romanzo manzoniano. Le poesie di Giorgio Vigolo ispirano Beniamino Mirisola, "La città dell'anima. Giorgio Vigolo tra psicanalisi e mito" (201-210), a esplorare le dialettiche tangenze fra psicanalisi, mondo onirico e metafore ossessive, e Varonica Tabaglio, "'Al centro delle visioni'. Una possibile lettura delle poesie di Giorgio 
Vigolo"(211-220), a riflettere sull'elemento autobiografico. Belli è il protagonista dei contributi di Marina Salvini, "Gli spettacoli popolari nei sonetti e nelle note di G.G.Belli” (59-68), sull'attenzione per il mondo del teatro e di Marialuigia Sipione, "Diabolus in Ecclesia? Le poesie religiose in lingua di G.G. Belli" (69-76) sulla Bibbia italiana dello scrittore romano. Elena Sbrojavacca, "Roberto Calasso e il ritorno degli Dei" (283-292), presenta la narrativa di Calasso e la "letteratura assoluta" (284), e infine Giulia Zava, "Interpretazione e ironia nelle immagini del Petrarca queriniano" (13-28), propone un'originale disamina sull'iconografia del Canzoniere miniato per mano del "Dilettante queriniano" (14). Chiude il volume l'Appendice dedicata ai quattro maestri di Gibellini: Isella, Contini ("Le Amicizie di Gianfranco Contini" 313-321), Segre ("Cesare Segre, maestro di rigore" 329330) e Corti ("Maria Corti: canti nel buio e segni luminosi” 323-327).

Loccasione di celebrazione del maestro si trasforma, dunque, in una vivace discussione su temi dissimili che trovano un'aggregante armonia nell'insegnamento di Gibellini "Ultimo dei Mohicani, testimone di un mondo (anche accademico) che non esiste quasi piư”.

Federica Conselvan

Università di Roma

Natalie Dupré, Monica Jansen, Srecko Jurisic e Inge Lanslots, a cura di. Narrazioni della crisi. Proposte italiane per il nuovo millennio. Firenze: Franco Cesati Editore, 2016. Pp. 162. ISBN 978-8-8766-7588-1.

Narrazioni della crisi è un libro al plurale su diversi fronti: quello editoriale della fitta co-curatela e dell'assiepamento dei contributi (quindici in poco più di centocinquanta pagine); quello geografico-culturale deducibile dai cognomi degli autori e dalle bibliografie di riferimento (manca purtroppo una scheda informativa sui collaboratori); quello metodologico dei diversi approcci al tema e, non ultimo, quello semantico della variegata interpretazione del concetto stesso di crisi. Se nei dintorni del Miracolo Economico Ennio Flaiano poteva scrivere "viviamo in un'epoca di transizione, come sempre del resto", il lettore traghettato dall'ultimo Novecento agli anni Zero non può che ritrovarsi nella premessa cardine dell'Introduzione al volume: "il vocabolo [crisi] ha invaso (...) in maniera capillare il piano esistenziale, al punto che ormai riesce difficile immaginare un mondo e una 